\title{
2DGE-coomassie brilliant blue staining used to differentiate pasteurized milk from reconstituted milk
}

\author{
Yajun $\mathrm{Wu}^{1}$, Ying $\mathrm{Chen}^{{ }^{1 *}}{ }^{*}$, Bin Wang${ }^{1}$, Haiyan Wang ${ }^{2}$, Fei Yuan ${ }^{1}$, Guiming Zhao ${ }^{1}$ \\ ${ }^{1}$ Chinese Academy of Inspection and Quarantine, No. 3, Gaobeidian North Street, Chaoyang District, Beijing, 100123, China \\ ${ }^{2}$ Internal Mongolia CIQ, No. 12, Erduosi Street, Huhehaote, 010020, China; yqychen@yahoo.com.cn
}

Received 27 October 2009; revised 4 September 2009; accepted 7 September 2009.

\begin{abstract}
Differentiating pasteurized milk and reconstituted milk by scientific approach was necessary to defend consumer from economic fraud of wrong labeling. In this paper 2DGE (2 Dimensional Gel Electrophoresis)-coomassie brilliant blue staining method was employed and significant color intensity changing was observed among raw milk, pasteurized milk, UHT milk and reconstituted milk. For example, the intensity of 10 protein spots including casein and lactoglobulin reduced more than two folds from pasteurized milk to reconstituted milk. However, DIGE (Differential Gel Electrophoresis) assay showed that the majority protein remained similar level from pasteurized milk to reconstituted milk. Therefore the color fading of coomassie brilliant blue stained 2D gels may be due to other biochemical reaction, such as Maillard reaction, instead of protein degradation. Stability of 2DGE pattern was confirmed by running six gels of the same sample in parallel and software analysis showed that all proteins were at similar level. Two commercialized pasteurized milk samples and one reconstituted milk sample were tested by 2DGE-coomassie blue staining method and reconstituted milk could be easily identified.
\end{abstract}

Keywords: 2DGE; Coomassie Brilliant Blue; Pasteurized Milk; Reconstituted Milk

\section{INTRODUCTION}

China has recently become one of the ten biggest milk producers in the world [1]. Fluid milk products include pasteurized milk and reconstituted milk. Chinese product standard of pasteurized milk (GB5408.1) commanded that only fresh raw milk could be used as raw material for

"Corresponding author. Tel: 0086-10-85783587; Fax: 0086-10-85774634 pasteurized milk, while reconstituted milk made by resolving milk powder in water and sterilization was categorized as sterilized milk (GB5408.2). Compared to pasteurized milk, reconstituted milk underwent more complicated thermal process including spray drying, pasteurization, UHT-treatment or in-bottle sterilization [2]. Because of higher cost at factory location, seasonal variation and transportation, pasteurized milk claims higher price than reconstituted milk. It is reported that the price for 8 tons of raw milk in China is about 20,000 YUAN, while the price for 1 ton of imported milk powder is $14,000-15,000$ YUAN which could be made into 8 tons of reconstituted milk [3]. However, as a lot of literature pointed out, intensive thermal treatment would compromise milk nutrition and flavor [4], thus consumers prefer pasteurized milk to reconstituted milk and were concerned at possible economic fraud by labeling reconstituted milk as pasteurized milk.

A few analysis techniques such as CE (Capillary Electrophoresis), HPLC (High performance liquid chromatography), ELSD (Evaporative Light-scattering Detector) have been applied in differentiating pasteurized milk and reconstituted milk [2,5]. In these methods, individual protein or sugar ingredient, for example furosine, lactoglobuline, HMF (hydroxymethylfurfural) is quantified, which demands complicated pre-procession of milk sample. Results of above-mentioned studies revealed significant change of protein component during the procession of milk. In this paper, we reported the application of 2DGE (2 Dimension Gel Electrophoresis) technique in an overall analysis of protein profile change related to milk thermal procession, revealing a significant alteration of protein component between pasteurized milk and reconstituted milk. Compared to other methods, 2DGE is characterized by simplicity in sample preparation, ability of parallel treatment of several samples and being information-rich. In recent years, 2DGE have been widely applied for food analysis [6-8]. A number of research work have been done in milk proteome such as Equidae milk [9], marsupial Trichosurus vulpecula milk [10], early lacatation milk of the tammar wallaby [11], $\kappa$-casein micro-heterogeneity in bovine milk [12] and whey protein [13]. In our proteomic 
study of milk product, it showed that 2D patterns after coomassie brilliant blue staining could differentiate pasteurized milk and reconstituted milk according to the change of color intensity of some protein spots.

\section{MATERIAL AND METHODS}

\subsection{Material}

Pooled raw milk sample was collected from Sanyuan Dairy Company and immediately sent to milk processing laboratory in Food Institute of China Agricultural University for heat processing. Dry milk powder was also collected from the company and reconstituted in accordance with the original milk/water ratio, then pasteurized. After preparation, total protein concentration of each sample was determined. Raw milk was centrifuged at $1100 \mathrm{~g}, 20 \mathrm{mins}$ and fat cream was removed. Three commercialized milk samples were bought from local supermarket including two pasteurized milk samples from different supplier and one reconstituted milk sample. All samples were stored at $4^{\circ} \mathrm{C}$ for immediate use or at $-80^{\circ} \mathrm{C}$.

\subsection{Total Protein Concentration Determination}

Total protein concentration was determined using Protein Assay Kit (NoVagen, Merk, Darmstadt, Germany) following the instruction. The optical absorbance value was recorded on ELISA reader (Thermo Fisher Scientific, MA, USA). Protein concentration was calculated on the basis of Absorbance-Concentration curve of reference BSA standards.

\subsection{DGE}

In preparation for IEF running, $10 \mu \mathrm{L}$ milk was mixed with $440 \mu \mathrm{L}$ of solubilization buffer consisting of $8 \mathrm{M}$ urea, 400mg/L CHAPS, 40 mM Tris, 50mg/L pH 4.7-5.9 carrier ampholytes (Bio-rad, Hercules, California, USA) and $100 \mathrm{mM}$ DTT. The sample was used to hydrate a $17 \mathrm{~cm}$ $\mathrm{pH}$ 4.7-5.9 IPG strip for $12 \mathrm{~h}$ at room temperature. Hydrated IPG strips were focused in a PROTEAN IEF System (Bio-rad, Hercules, California, USA) at $100 \mathrm{~V}$ for $1 \mathrm{~h}$ followed by $500 \mathrm{~V}$ for $1 \mathrm{~h}$ and $1 \mathrm{kV}$ for $1 \mathrm{~h}$ before the voltage was increased to $8 \mathrm{kV}$ for a total of $100 \mathrm{kVh}$. In the second dimensional SDS-PAGE assay, focused strips were first balanced in equilibrium buffer I and buffer II, then embedded with $0.5 \%$ agarose on top of $14 \%$ polyacrylamide gels $(18 \times 18 \mathrm{~cm})$. Electrophoresis was performed in PROTEAN II XL Cell (Bio-rad, Hercules, California, USA) at $5 \mathrm{~mA} / \mathrm{gel}$ for $2 \mathrm{~h}$ followed by 20 $\mathrm{mA} /$ gel for $16 \mathrm{~h}$. Gels were stained with Coomassie Brilliant Blue G-250 and destained in 1\% acetic acid. Images were captured on Versadoc Imager (Bio-rad, Hercules, California, USA) in transmission mode.

\subsection{DIGE}

Milk samples were labeled with Cy dye (CyDye DIGE Fluors, GE Healthcare, Buckinghamshire, UK) according to the instruction. Sample pooling strategy was modified as $5 \mu \mathrm{L}$ milk labeled with $1 \mu \mathrm{L}$ cy working solution $(400 \mathrm{pmol} / \mu \mathrm{L})$. All of the $10 \mu \mathrm{L}$ labeled sample comprised with $5 \mu \mathrm{L}$ pasteurized milk and $5 \mu \mathrm{L}$ reconstituted milk was pooled together. 2DGE was run following abovementioned procedure.

\subsection{Data Analysis}

2DGE profiles caught by Versadoc imager were analyzed with PDQuest software 7.4.0 (Bio-rad, Hercules, California, USA). After automatic spot detection, spot view was performed to display quantity of protein spots. For DIGE imaging, specific cy channel was selected.

\subsection{Spot Digestion}

In-gel digest was conducted following procedure of literature (Holland etal. 2004). Digestion product was purified using Ziptip $\mathrm{C}^{18}$ pipette tip (Millipore, Danvers, MA, USA) following instruction. In the final elution step, peptide was dissolved in $10 \mathrm{mg} / \mathrm{mL}$ a-cyano-4-hydroxycinnamic acid in $0.1 \% \mathrm{TFA} / 50 \% \mathrm{ACN}$ and directly applied to MALDI-TOF analysis.

\subsection{MALDI-TOF and Database Search}

One microliter purified peptide solution was spotted onto a stainless steel MALDI target. Spectra were acquired using a 4700 MALDI-TOF mass spectrometer (Applied Biosystems, Foster City, CA, USA) in delayed extraction mode. Tryptic digests were analyzed in positive ion reflectron mode with an accelerating voltage of $20 \mathrm{kV}$, grid voltage at $64 \%$ and a delay time of 165 ns. One hundred laser shots were accumulated for each spectrum. Peptide mass fingerprint (PMF) of cut protein spot was analyzed by MS-fit program of the ProteinProspector software (University of California, USA). SwissProt.20071010 database was searched and searching parameter was set as follow: Bos Taurus species, Tol $1 \mathrm{Da}$, Min matched peptide set as 6 .

\section{RESULTS AND DISCUSSION}

Raw milk sample was taken from pooled milk container to minimize heterogeneity of protein composition. Milk was processed in lab to ensure the authenticity of processing condition. Different PI ranges were tried to determine the best 2DGE condition (2DGE of methods section). As shown in Figure 1, pH4.9-5.7 IPG strip produced the most satisfied 2D pattern in terms of protein spot quantity and separating size among these spots.

Total protein concentration of raw milk, pasteurized milk, UHT milk and reconstituted milk was calculated by Biuret method as shown in Table 1. After comparison of the 2D-coomassie brilliant blue staining patterns of the four milk samples shown in Figure 2, we found that for the majority of protein spots, color intensity decreased si gnificantly from raw milk, pasteurized milk, UHT milk to 


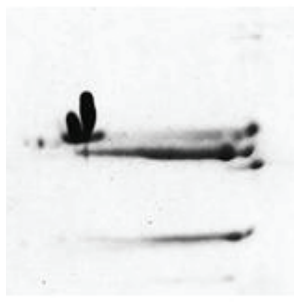

pH3.9-5.1

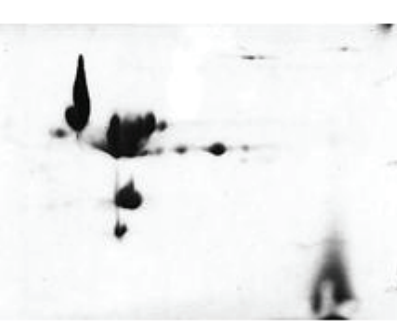

pH4-7

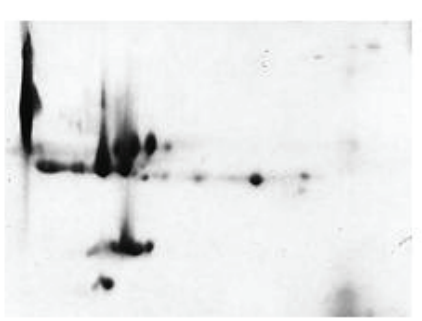

pH4.9-5.7

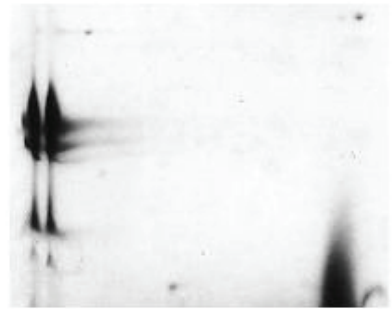

pH5.5-6.7

SDS-PAGE was run in $7 \mathrm{~cm} \times 7 \mathrm{~cm}$ gels.

Figure 1. 2DGE profiles at different IPG range.

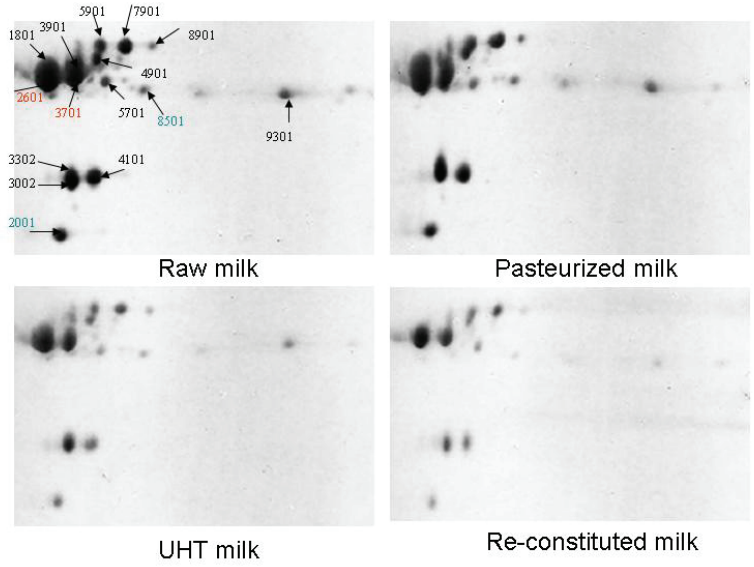

Ten microliter milk of each sample the protein concentration of which has been modulated at the same level was loaded.

Figure 2. 2DGE profiles of raw milk, pasteurized milk, UHT milk and reconstituted milk.

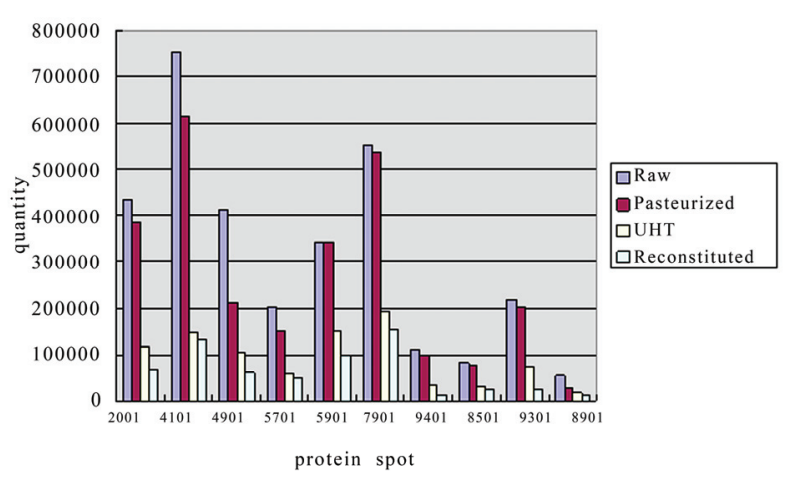

Those spots were begot by PDQuest software analysis. The analysis set manager was defined as pasteurized milk two times higher in quantity than reconstituted milk.

Figure 3. Quantitative comparison of ten protein spots among raw milk, pasteurized milk, UHT milk and reconstituted milk.

reconstituted milk. Quantitative analysis by PDQuest confirmed the trend. As shown in Figure 3, after the analysis set template was defined as pasteurized milk being two fold above reconstituted milk, ten spots were
Table 1. Protein concentration of raw milk, pasteurized milk, UHT milk and reconstituted milk.

\begin{tabular}{ccccc}
\hline Assay & \multicolumn{4}{c}{ Raw milk Pasteurized milk UHT milk Reconstituted milk } \\
\hline A1 & 0.227 & 0.237 & 0.233 & 0.228 \\
A2 & 0.224 & 0.235 & 0.227 & 0.231 \\
A3 & 0.23 & 0.224 & 0.24 & 0.225 \\
$A$ & 0.227 & 0.232 & 0.233 & 0.228 \\
C(mg/ml) & 23.5 & 23.8 & 23.8 & 23.6 \\
\hline
\end{tabular}

detected. The quantity of the protein spots as represented by the $\mathrm{Y}$ axis decreased when milk was heat treated and the difference between pasteurized milk and reconstituted milk was remarkable.

In order to prove that under standard operation procedure 2DGE pattern was characterized by good repeatability, six 2D gels of pasteurized milk were run in parallel. Raw maps of coomassie blue staining were shown in Figure 4. All gels presented similar pattern and PDQuest analysis showed similar quantity level for all protein spots.

Protein spots were extracted, digested and identified by MALDI-TOF. As shown in Figure 5, 16 spots were successfully identified as casein and its isomers, lactoglobulin and lactate dehydronese-like protein.

The above experiments demonstrated that after coomassie blue staining, color intensity of most proteins decreased when milk sample was processed under thermo condition and the difference between pasteurized milk and reconstituted milk was significant enough to be used in product identification. As for the reason behind the changing trend, Maillard reaction should be considered other than proteins degradation since Maillard was the most significant biochemical process during heat treatment of milk $[4,14]$. In Maillard reaction, the reducing sugar covalently binds to the epsilon amide residue of lysine. We inferred that the intensity decrease of protein spots was related to coomassie brilliant blue staining. DIGE (2D Difference Gel Electrophoresis) was then conducted to understand the true situation of protein 

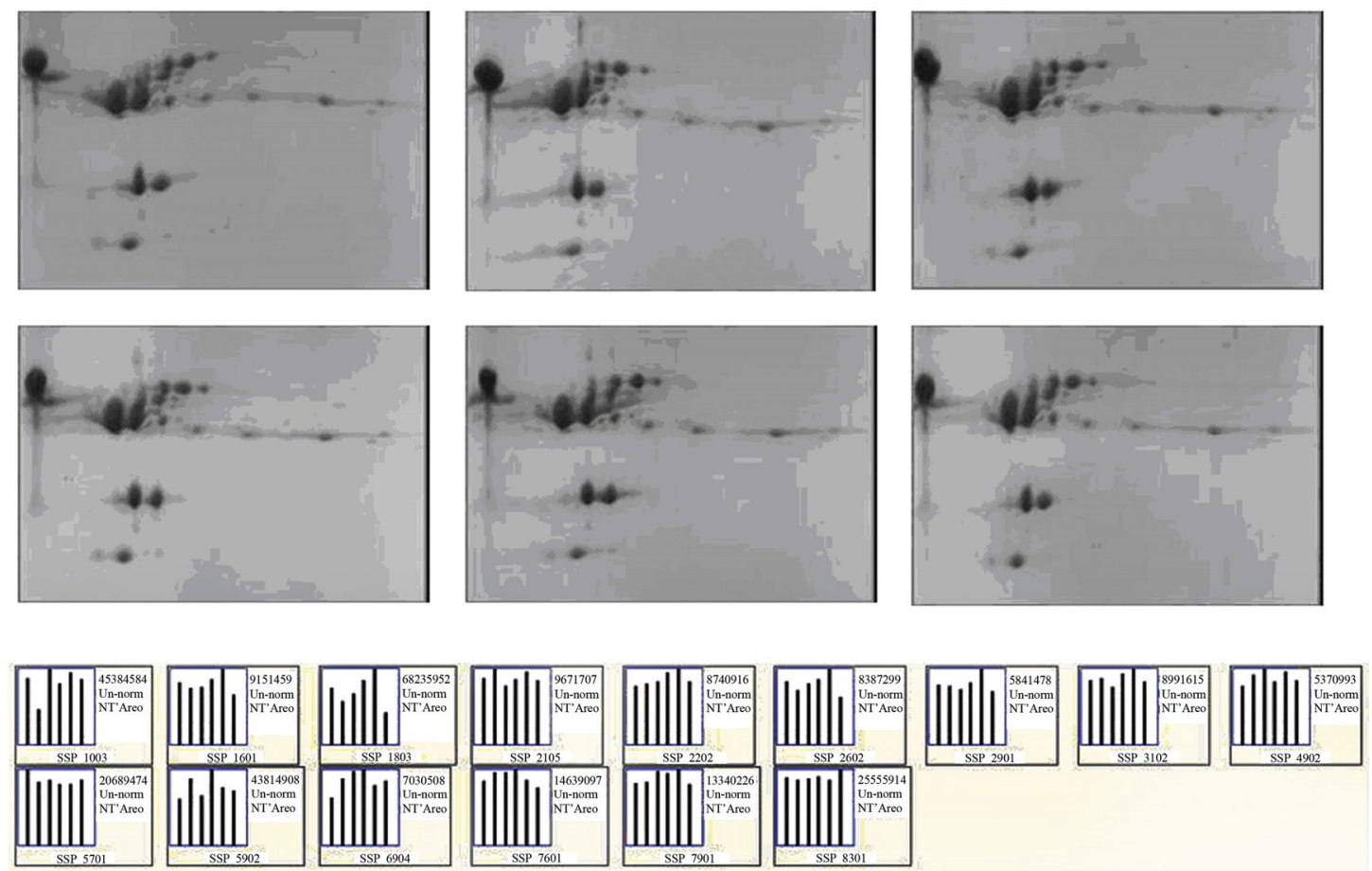

2DGE profile was shown as raw map. Spot quantity was shown in the small box below the map.

Figure 4. Six 2DGE profiles of pasteurized milk run in parallel to confirm the stability of the method.

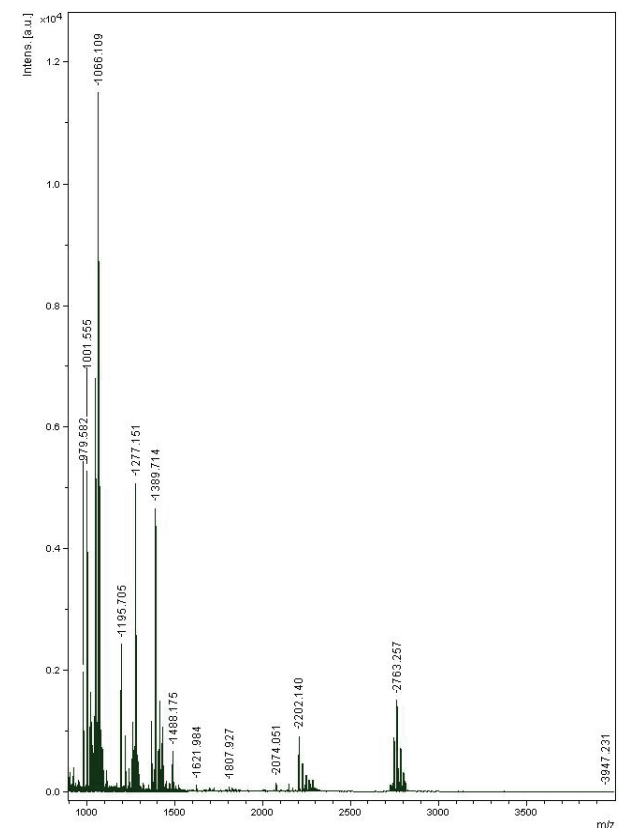

\begin{tabular}{c|l} 
SSP ID & Protein function \\
\hline 3701 & Beta-casein \\
5901 & Alpha-S2-casein \\
4901 & Alpha-S2-casein \\
7901 & Alpha-S2-casein \\
8901 & Alpha-S2-casein \\
5701 & L-lactate dehydrogenase A-like 6B \\
9401 & Kappa-casein \\
3302 & Bate-lactoglobulin \\
4101 & Bate-lactoglobulin \\
1701 & L-lactate dehydrogenase A-like 6B \\
4701 & L-lactate dehydrogenase A-like 6B \\
6901 & Alpha-S2-casein \\
6801 & Alpha-S2-casein \\
7601 & L-lactate dehydrogenase A-like 6B \\
3001 & Alpha-S1-casein \\
3903 & Alpha-S2-casein
\end{tabular}

The left figure was peptide mass fingerprint of one protein after in-gel digestion. The right table showed identification results of several proteins after database search.

Figure 5. Identification of milk proteins by MALDI-TOF.

quantity changing between pasteurized milk and reconstituted milk. As shown in Figure 6, after pre-stained with cy3 and cy5 respectively, pasteurized milk and reconsti- tuted milk were mixed together and run in the same 2D gel. Images of cy3 and cy5 showed that the majority of protein spots had similar level of fluorescence intensity. 


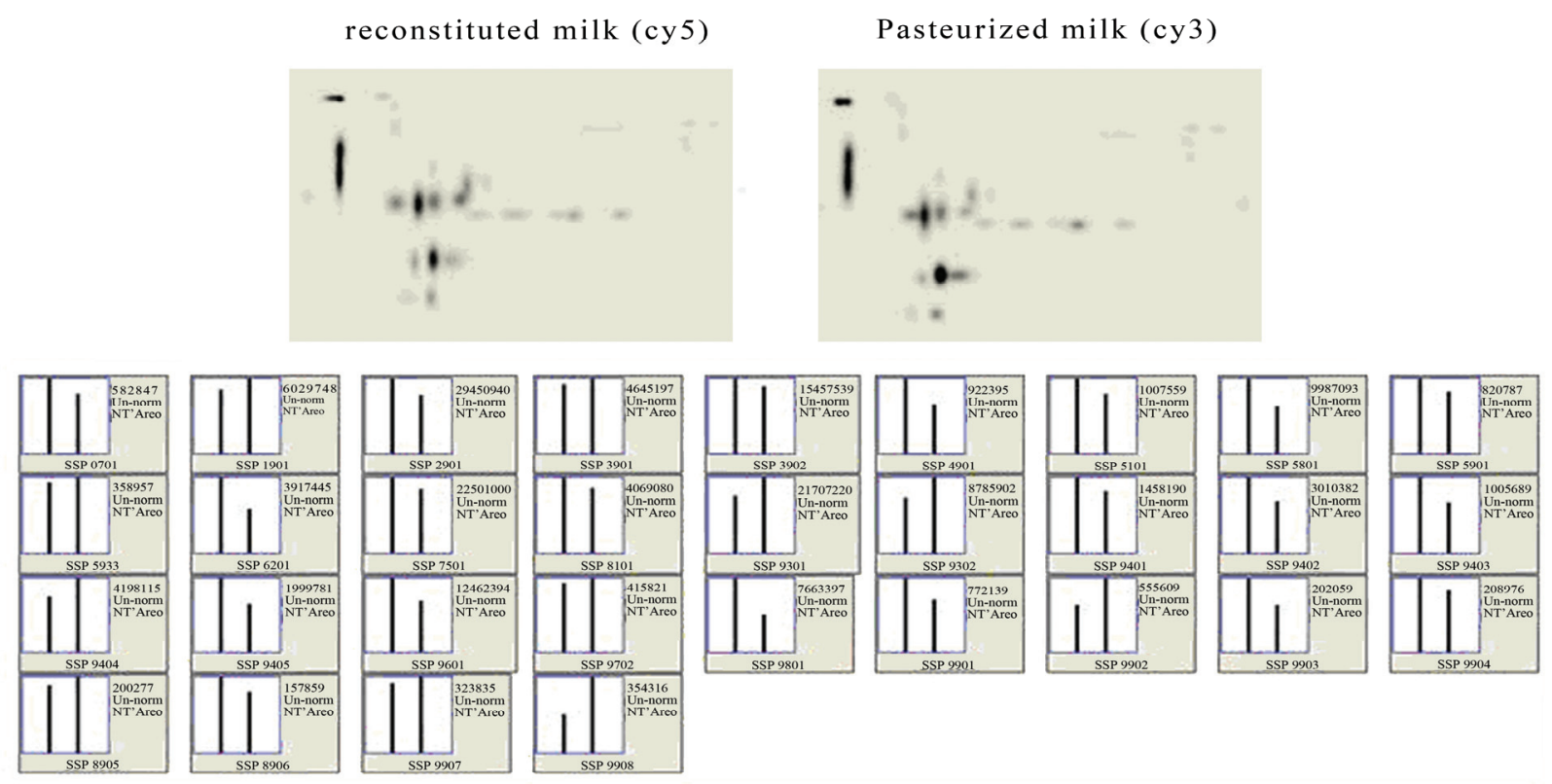

Profiles were shown as Gaussian map. Spot quantity was shown in the small box below the map. In each box, left column corresponded to reconstituted milk and right column to pasteurized milk.

Figure 6. DIGE profiles of pasteurized milk and reconstituted milk.

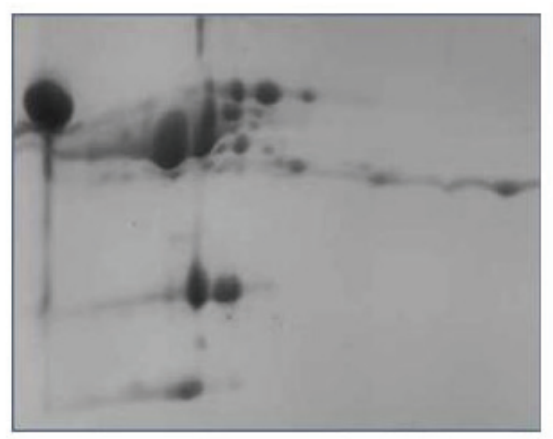

\section{Reference \\ Pasteurized milk}

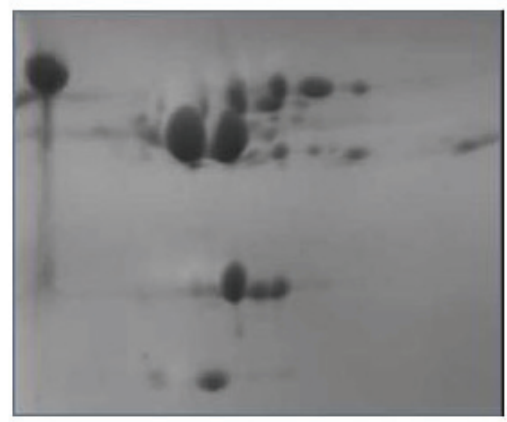

Sample2

pasteurized millk

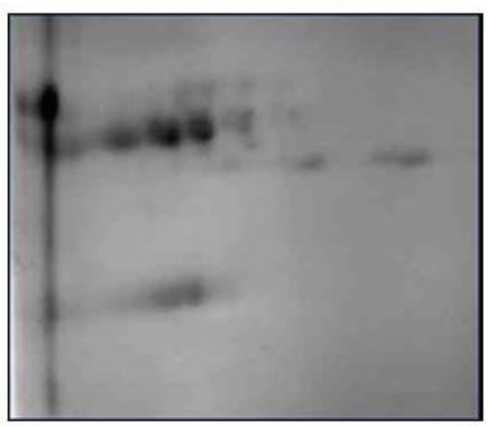

Sample3 reconstituted milk

These three samples were bought from local supermarket.

Figure 7. 2DGE-coomassie brilliant blue staining profiles of three commercialized milk samples. 
PDQuest analysis indicated that the quantity difference between pasteurized milk and reconstituted milk was less than two folds. Thus we suppose the remarkable diverge between cydye labeling and coomassie blue staining was due to different staining mechanism. Cydye DIGE fluors were designed to covalently attach to the epsilon amino group of lysine of proteins in a "minimal labeling" way which means the dyes labeled only on a single lysine per protein molecule. Therefore Maillard reaction would not affect staining efficiency. As for coomassie blue, the mechanism of staining is still not well understood ever since it was used in protein staining 45 years ago. However some literatures proved that coomassie blue varied widely in its ability to bind proteins due to its affinity for protein rich in basic amino acids such as lysine, arginine and histidine and its poor ability to bind with glycoprotein [15]. Moreover, since coomassie blue staining was not a "minimal labeling" technique, its labeling efficiency would be affected significantly by Maillard reaction. In fact the content of available lysine has often been used as marker of heat damage affecting dairy protein [16].

In order to verify the practicality of the method, three commercialized milk samples plus the reference pasteurized milk sample were tested in parallel. As shown in Figure 7, two pasteurized milk samples resulted in similar 2D pattern as reference sample. The reconstituted milk sample showed remarkably different 2D pattern. The seriously reduced level of protein staining indicated that the reconstituted milk sample might undergo stronger thermo treatment than pasteurization.

Therefore, 2DGE-coomassie brilliant blue staining technique was proved to be very useful in differentiation between pasteurized milk and reconstituted milk in that color intensity of most proteins decreased significantly after heat treatment. DIGE experiment showed that true quantity of these proteins did not change much. Change of color intensity of different samples may be due to the decrease of labeling efficiency of coomassie blue caused by Mailard reaction after heat treatment. Therefore we could apply 2DGE-coomassie brilliant blue staining method for identification of pasteurized milk and reconstituted milk. Moreover 2DGE could be used to detect adulterated milk product according to different $2 \mathrm{D}$ pattern of protein from different organism or tissue.

\section{ACKNOWLEDGEMENT}

This work was supported by the CAIQ (China Academy of Inspection and Quarantine) Research Fund (2007JK005) and the Eleventh Five-year Plan Research Fund of the Ministry of Science and Technology, People's Republic of China (2006BAD27B02). We greatly appreciate the kindness of professor Ren Zhenfa of Chinese Agriculture University in helping with sample preparation.

\section{REFERENCES}

[1] A. Fagerberg, (2008) Field study on milk quality in China, Internatinal Dairy Topics, 7(1), 11-12.

[2] J. D. Block, M. Merchiers, L. Mortier, A. Braekman, W. Ooghe, R. V. Renterghem, (2003) Monitoring nutritional quality of milk powders: capillary electrophoresis of the whey protein fraction compared with other methods, International Dairy Journal, 13, 87-94.

[3] G. X. Liu, (2003) Reconstituted milk $\neq$ Pure fresh milk, City Quality Supervision, 4, 46.

[4] M. Lacroix, J. Leonil, C. Bos, G. Henry, G. Airinei, J. Fauquant, D. Tome, C. Gaudichon, (2006) Heat markers and quality indexes of industrially heat-treated $\left[{ }^{15} \mathrm{~N}\right]$ milk protein measured in rats, J. Agric. Food Chem., 54, 1508-1517.

[5] F. Guyomarch, F. Warin, D. Muir, J. Leave, (2000) Lactosylation of milk proteins during the manufacture and storage of skim milk powders, International Dairy Journal, 10, 863-872.

[6] M. Carbonaro, (2004) Proteomics: present and future in food quality evaluation, Trends in Food Science \& Technology, 15, 209-216.

[7] M. A. Manso, J. Leonil, G. Jan, V. Gagnaire, (2005) Application of proteomics to the characterization of milk and dairy products, International Dairy Journal, 15, 845-855.

[8] R. O. Donnella, J. W. Hollanda, H. C. Deethb, P. Alewooda, (2004) Milk proteomics, International Dairy Journal, 14, 1013-1023.

[9] G. Miranda, F. M. Mahé, C. Leroux, P. Martin, (2004) Proteomic tools to characterize the protein fraction of Equidae milk, Proteomics, 4, 2496-2509.

[10] S. Kuy, V. C. Kelly, A. M. Smit, D. J. Palmer, G. J. Cooper, (2007) Proteomic analysis of whey and casein proteins in early milk from the marsupial Trichosurus vulpecula, the common brushtail possum, Comparative Biochemistry and Physiology, 2, 112-120.

[11] J. Joss, M. Molloy, L. Hinds, E. Deane, (2007) Proteomic analysis of early lactation milk of the tammar wallaby (Macropus eugenii), Comparative Biochemistry and Physiology, 2, 150-164.

[12] J. W. Holland, H. C. Deeth, P. F. Alewood, (2004) Proteomic analysis of k-casein micro-heterogeneity, Proteomics, 4, 743-752.

[13] C. J. Hogarth, J. L. Fitzpatrick, A. M. Nolan, F. J. Young, A. Pitt, P. D. Eckersall, (2004) Differential protein composition of bovine whey: A comparison of whey from healthy animals and from those with clinical mastitis, Proteomics, 4, 2094-2100.

[14] P. Resmini, L. Pellegrino, F. Masott, A. Tirelli, F. Prati, (1992) Detection of reconstituted milk powder in raw and in pasteurized milk by HPLC of furosine, Scienzae Tecnica Lattiero Casearia, 3(3), 169-186.

[15] K. McDonald, (2009) Overcoming the Coomassie blues. Drug Discovery \& Development.

http://www.dddmag.com/Article-Overcoming the Coomas-Sie Blues-060409.aspx

[16] S. M. Rutherfurd, P. J. Moughan, (1997) Application of a new method for determining digestible reactive lysine to variably heated protein sources, J. Agric. Food Chem., 45(5), 1582-1586. 